\title{
Profissionais a exercer na Medicina Dentária: Principais Fatores de Risco e Riscos Laborais, Doenças Profissionais Associadas e Medidas de Proteção Recomendadas
}

rpso.pt/profissionais-exercer-na-medicina-dentaria-principais-fatores-risco-riscos-laborais-doencas-profissionaisassociadas-medidas-protecao-recomendadas/

DENTAL MEDICINE WORKERS: MAIN LABOR FACTOR RISKS AND RISKS, ASSOCIATED PROFESSIONAL DISEASES AND

RECOMMENDED PROTECTIVE MEASURES

TIPO DE ARTIGO: Revisão Bibliográfica Integrativa

AUTORES: Santos M(1), Almeida A(2), Oliveira T(3).

\section{RESUMO}

\section{Introdução/ enquadramento/ objetivos}

Nas instituições prestadoras de serviços dentários existem geralmente médicos dentistas (generalistas e/ou especializados), os ajudantes/ auxiliares dentários, rececionistas e, por vezes, gestores/ orçamentistas.

Os principais fatores de risco/ riscos laborais dos primeiros são o eventual contato com agentes biológicos; posturas mantidas/ forçadas, movimentos repetitivos e vibrações; ruido; radiações eletromagnéticas e esforço visual por utilizarem écrans com frequência; radiação ionizante por $\mathrm{Rx}$ e agentes químicos. As restantes classes profissionais atrás mencionadas estão sujeitas a radiações eletromagnéticas, esforço visual, postura sentada mantida e movimentos repetitivos. Existem uma quantidade razoável de trabalhos publicados relativos à saúde ocupacional neste setor, mas a generalidade destes aborda aspetos específicos, não sendo fácil encontrar uma perspetiva global do tema, minimamente completa e sucinta. Pretende-se com esta revisão conhecer melhor os fatores de risco/ riscos existentes neste setor profissional.

\section{Metodologia}


Trata-se de uma Revisão Bibliográfica Integrativa, iniciada através de uma pesquisa realizada em dezembro de 2017 nas bases de dados "CINALH plus with full text, Medline with full text, Database of Abstracts of Reviews of Effects, Cochrane Central Register of Controlled Trials, Cochrane Database of Systematic Reviews, Cochrane Methodology Register, Nursing and Allied Health Collection: comprehensive, MedicLatina, Academic Search Complete e RCAAP".

\section{Conteúdo}

Os agentes biológicos mais relevantes neste contexto são os vírus das hepatites B e C, bem como o HIV.

Em diversas tarefas estes profissionais permanecem por períodos prolongados em posturas mantidas e/ou forçadas (quer sentados, quer de pé e quase estáticos, ainda que com alguns movimentos repetitivos associados e, por vezes, sem pausas adequadas).

Neste setor profissional há exposição relevante ao ruído. Os instrumentos de trabalho que apresentam níveis mais elevados de decibéis foram a turbina de alta rotação e o compressor; também foram mencionados as canetas odontológicas, o compressor de ar, o aspirador, o amalgamador e os próprios aparelhos de climatização.

De todos os agentes químicos usados até agora, o mercúrio é o mais relevante.

Em alguns contextos há exposição a Rx.

\section{Conclusões}

Nenhum dos artigos selecionados mencionou de forma direta medidas de proteção coletiva. Contudo, poder-se-ão destacar neste contexto o uso de agentes químicos o menos tóxicos possíveis, um bom desenho ergonómico do posto de trabalho, utilização de equipamentos que emitam o menor ruído e vibração possível, rotatividade de tarefas e formação adequada aos profissionais relativa aos fatores de risco e riscos laborais.

A nível de equipamentos de proteção individual são mencionados o uso de bata de manga comprida, viseira ou óculos, gorro e luvas de latex.

Os acidentes de trabalho neste setor justificam-se sobretudo pelo campo de trabalho pequeno, uso de instrumentos cortantes e/ou que trabalham a alta velocidade, sendo razoavelmente frequente a criação de aerossóis e salpicos, bem como pela proximidade física com o cliente e/ou os movimentos bruscos que este possa ter. A parte do corpo geralmente mais atingida com acidentes por corto-perfurantes é o dedo; as agulhas nunca devem ser recapsuladas com a outra mão a segurar a tampa, nem as agulhas devem ser reutilizadas, encurvadas ou partidas.

Nenhum dos artigos consultados deu particular destaque para as doenças profissionais; contudo, são de realçar a este nível a patologia músculo-esquelética variada, doenças infeciosas (HIV, hepatites B e C, sobretudo), questões oncológicas diversas e, ao nível 
emocional, ansiedade, depressão e burnout.

Seria pertinente desenvolver investigações que avaliassem a realidade nacional.

PALAVRAS/ EXPRESSÕES- CHAVE: saúde ocupacional, saúde do trabalhador e medicina do trabalho; dentista; assistente dentário.

\section{ABSTRACT}

\section{Introduction / objectives}

In dental care institutions there are usually dentists (general practitioners and/ or specialists), dental assistants, receptionists and sometimes managers.

The main risk factors/ occupational risks of the former are the possible contact with biological agents; maintained/ forced postures, repetitive movements and vibrations; noise; electromagnetic radiation and visual effort to use screens with frequency; ionizing radiation by $\mathrm{Rx}$ and chemical agents. The other professional classes mentioned above are subject to electromagnetic radiation, visual effort, maintained seated posture and repetitive movements. There are a fair amount of published work on occupational health in this sector, but the generality of these addresses specific aspects, and it is not easy to find a global perspective of the subject, minimally complete and succinct. This review intends to better understand the risk/ risk factors in this professional sector.

\section{Methodology}

This is an Integrative Bibliographic Review, initiated through a survey conducted in December 2017 in the databases "CINALH plus with full text, Medline with full text, Database of Abstracts of Reviews of Effects, Cochrane Central Register of Controlled Trials, Cochrane Database of Systematic Reviews, Cochrane Methodology Register, Nursing and Allied Health Collection: comprehensive, MedicLatina, Academic Search Complete and RCAAP “.

\section{Content}

The most relevant biological agents in this context are hepatitis $B$ and $C$ viruses, as well as HIV.

In several tasks these professionals remain for prolonged periods in maintained and/ or forced postures (sitting, standing and almost static, although with some repetitive movements associated and sometimes without adequate pauses).

In this professional sector there is relevant exposure to noise. The instruments with higher decibel levels were the high-speed turbine and the compressor; dental pens, the air compressor, the vacuum cleaner, the amalgamator and the air conditioning units themselves.

Of all the chemical agents used so far, mercury is the most relevant. 
In some contexts there is exposure to Rx.

\section{Conclusions}

None of the selected articles mentioned collective protection measures directly. However, in this context, the use of the least toxic chemical agents, a good ergonomic design of the work station, the use of equipment that emits the least possible noise and vibration, the rotation of tasks and the appropriate training of professionals.

At the level of personal protective equipment are mentioned the use of long-sleeved gown, visor or glasses, hat and gloves of latex.

Work accidents in this sector are mainly justified by the small work field, the use of sharp instruments and/ or high speed work, with the creation of aerosols and splashes being fairly frequent, as well as physical proximity to the client and/ or the sudden movements that it may have. The part of the body generally most affected by short-piercing accidents is the finger; the needles should never be recapsulated with the other hand holding the cap, nor should the needles be reused, bent or broken.

None of the articles consulted focused on occupational diseases; however, a variety of musculoskeletal pathologies, infectious diseases (HIV, hepatitis B and C, above all), various oncological issues, and emotional level, anxiety, depression and burnout are highlighted at this level.

It would be pertinent to develop investigations that evaluate the national reality.

WORDS I KEY EXPRESSIONS: occupational health, worker health, and occupational medicine; dentist; dental assistant.

\section{INTRODUÇÃO}

Os principais fatores de risco/ riscos laborais dos médicos dentistas são o eventual contato com agentes biológicos relevantes por corte/ perfuração cutânea, inalação ou contato por mucosa (e infeções associadas); posturas mantidas/ forçadas, movimentos repetitivos e vibrações (e consequentes lesões músculo-esqueléticas); ruido (e eventual hipoacusia); radiações eletromagnéticas (com consequências não consensuais na literatura) e esforço visual por utilizarem écrans com frequência (e eventual cansaço, perda de acuidade); radiação ionizante por Rx (e possíveis consequências cancerígenas); agentes químicos (e eventuais patologias oncológica, neurológica, dermatológica, respiratória e otorrinolaringológica, sobretudo), bem como stress (em função da personalidade e capacidade de coping do funcionário, com eventuais depressão/ burnout associados).

Por sua vez, as restantes classes profissionais atrás mencionadas estão sujeitas a radiações eletromagnéticas, esforço visual, postura sentada mantida e movimentos repetitivos a nível de rato/ teclado. 
Existem uma quantidade razoável de trabalhos publicados relativos à saúde ocupacional neste setor, mas a generalidade destes aborda aspetos específicos, não sendo fácil encontrar uma perspetiva global do tema, minimamente completa e sucinta.

\section{METODOLOGIA}

Pergunta protocolar: quais os principais riscos e fatores de risco existentes para os profissionais que trabalham na Medicina Dentária, eventuais doenças profissionais associadas e medidas de proteção recomendadas?

Em função da metodologia PICo, foram considerados:

-P (population): funcionários a exercer em clínicas dentárias.

-I (interest): reunir conhecimentos relevantes sobre os principais riscos e fatores de risco existentes para os profissionais que exercem na Medicina Dentária, eventuais doenças profissionais associadas e medidas de proteção recomendadas, segundo os dados mais recentemente publicados

-C (context): saúde ocupacional nas empresas deste setor

Foi realizada uma pesquisa em dezembro de 2017 nas bases de dados "CINALH plus with full text, Medline with full text, Database of Abstracts of Reviews of Effects, Cochrane Central Register of Controlled Trials, Cochrane Database of Systematic Reviews, Cochrane Methodology Register, Nursing and Allied Health Collection: comprehensive, MedicLatina e Academic Search Complete". Utilizando as palavras-chave "dentist" foram obtidos 1493 artigos; conjugando com "occupational" foram obtidos 74 trabalhos, com os critérios publicação igual ou superior a 2007 e acesso a texto completo; foram selecionados após a leitura do resumo 23 artigos e, após a consulta do trabalho na íntegra, 20 artigos.

Contudo, como não se encontraram estudos relativos à realidade portuguesa nestas bases de dados indexadas, os autores procuraram trabalhos inseridos no RCAAP (Repositório Científico de Acesso Aberto em Portugal). Aqui, utilizando a palavra "dentista", foram obtidos 1196 documentos; optou-se por conjugar com o termo "ocupacional" e aí já se reduziu a amostra para 10 trabalhos; após a leitura do resumo dos mesmos foram selecionadas 5 investigações; após a consulta na íntegra selecionaram-se 3 artigos.

O resumo da metodologia aplicada nesta revisão pode ser consultado nos fluxogramas de $1^{\mathrm{a}}$ e $2^{\mathrm{a}}$ fases.

\section{CONTEÚDO}

\section{Agentes Biológicos}

Os agentes biológicos mais relevantes neste contexto são os vírus das hepatites $\mathrm{B}$ e $\mathrm{C}$, bem como o HIV ${ }^{1-3}$, com riscos médios de infeção de 3 a 10, 10 e 0,2 a 0,3\%, respetivamente; contudo, na generalidade das populações, os primeiros são muito mais 
prevalentes $^{1}$.

A maioria dos dentistas experiencia, pelo menos, um episódio de lesão com instrumentos corto-perfurantes, previamente utilizados no paciente. Aliás, num estudo Tailandês, $23 \%$ referiu ter uma situação destas pelo menos semanalmente ${ }^{1}$.

O risco aparenta ser mais relevante nos profissionais com mais idade, mais anos de experiência profissional, menor adesão às normas existentes e menores conhecimentos acerca dos microrganismos e patologias associadas ${ }^{1}$; por vezes, até negando cuidados médicos a pessoas infetadas, segundo relatos de alguns países menos desenvolvidos ${ }^{4}$.

A luva de latex, ainda que não impeça a entrada da agulha, pode atenuar até $70 \%$ a quantidade de sangue presente. A saliva comporta um risco menor, mesmo com vestígios hemáticos. A lavagem da lesão diminuiu o número de microrganismos, mas o esfregar vigoroso pode facilitar a entrada dos mesmos ${ }^{2}$.

O não cumprimento com os protocolos existentes pode estar associado ao receio de intimidar/ colocar o paciente numa posição desconfortável ${ }^{2}$. Por vezes, na experiência clínica dos autores, tal também pode acontecer porque o profissional considera que o risco de infeção é quase nulo ou entender que o processo seria uma perda de tempo e/ou considerar que as medidas protocoladas não são eficazes.

A quimioprofilaxia para o HIV deverá ser iniciada até duas horas; no caso da hepatite B, a gamaglobulina deverá ser administrada até 48 horas $^{2}$, segundo alguns autores.

\section{Posturas forçadas/ mantidas, movimentos repetitivos e vibrações}

Dentistas e ortodentistas (especialistas na correção da posição dos dentes) apresentam níveis elevados de patologia músculo-esquelética ${ }^{5-7}$.

Em diversas tarefas estes profissionais permanecem por períodos prolongados em posturas mantidas e/ou forçadas ${ }^{6,8-10}$ (quer sentados, quer de pé e quase estáticos ${ }^{11}$, ainda que com alguns movimentos repetitivos associados e, por vezes, sem pausas adequadas $)^{5,11}$. Para além disso, podem também ocorrer iluminância desadequada ${ }^{5,6}$, mau posicionamento do paciente, stress, má aptidão física e/ou idade mais avançada ${ }^{6}$. Para agravar a situação, várias tarefas implicam compressão mecânica nas mãos ${ }^{5}$.

Quanto mais prolongados são os turnos ${ }^{6,7}$ e quanto mais idade/ tempo de exposição aos riscos laborais existe, mais prováveis serão as LMEs (lesões músculo-esqueléticas). Para além disso, tarefas que recrutam menos músculos e/ou músculos mais pequenos, a sensação de fadiga pode ficar potenciada ${ }^{6}$.

Um artigo alemão quantificou, por exemplo, que cerca de $81 \%$ dos dentistas avaliados referiam cervicalgias e omalgias ${ }^{11}$; outros estudos mencionam quantificações que vão até $90 \%{ }^{6,9}$. Numa investigação brasileira quantificou-se que $48 \%$ dos profissionais inseridos na amostra estudada apresentavam lesão por esforço repetitivo, sobretudo nos 
ombros, coluna, mãos, punhos, cotovelos e dedos (por ordem decrescente, respetivamente); contudo, só considerando o sexo feminino, no global, tal valor subia para $77 \% 5$.

A disposição ergonómica do profissional e do paciente adquirem relevância fundamental na prevenção e atenuação das LMEs, através da manutenção da postura neutra ${ }^{11}$.

Assim, para além das caraterísticas do trabalho (como repetição, posturas forçadas/ mantidas e vibrações), também contribuem para o surgimentos destas patologias questões individuais (como sexo, idade, altura e peso) e questões psicossociais (carga de trabalho exigida, personalidade e atitudes de todos os envolvidos) ${ }^{6}$.

Alguns investigadores concluíram que os ortodentistas têm queixas mais frequentes a nível das mãos/punhos, enquanto que os dentistas generalistas referem mais frequentemente omalgias. Alguns defendem que quanto mais especializada é a tarefa, mais frequentes se tornam os sintomas ${ }^{6,7}$.

Numa fase inicial os sintomas surgem apenas durante o trabalho e desaparecem no repouso; posteriormente mantêm-se mesmo nessa fase e aqui já se nota geralmente diminuição da capacidade de trabalho ${ }^{12}$.

A postura ideal do dentista é aquela que melhor acesso lhe dá à boca do paciente, mantendo-se simultaneamente o conforto do profissional. Contudo, ainda que o tema seja sempre abordado na formação académica dos mesmos, nem todos conseguem transpor para a sua prática clínica tais noções. Por vezes a postura é errada devido ao hábito/ rotina e/ou a ambientes de trabalho mal desenhados. Na generalidade dos gabinetes teremos a considerar o cliente deitado, a iluminação, o carrinho móvel, o sistema de vácuo e os pedais de controlo, para além de eventuais peças fixas de mobiliário, além da posição assumida pelo assistente dentário ${ }^{13}$.

A postura sentada neutra carateriza-se por ser natural, simétrica, com tensão muscular mínima e estável. A região dorsal deverá permanecer retilínea e não curva; a inclinação anterior do tronco não deverá ser superior a 20\%; a inclinação anterior da cabeça não deverá sair dos $20-25^{\circ}$ em relação ao tronco; os membros superiores deverão estar próximos do tronco, com orientação a cerca de $10^{\circ}$ e com os antebraços levantados sensivelmente a $25^{\circ}$ em relação à linha horizontal; o ângulo entre as ancas/ coxas e os pés deverá ser superior a $105^{\circ}$; as coxas devem ficar afastadas cerca de $45^{\circ}$; os pés devem permanecer paralelos ao chão e discretamente posteriores e simétricos ${ }^{13}$.

Idealmente, a superfície do dente a tratar deverá estar paralela ao dentista, com vista perpendicular, a uma distância de 35 a 40 centímetros, ou um pouco mais se o dentista for alto.

Nem uma postura correta exige uma manutenção rígida da mesma; ou seja, há liberdade para fazer movimentos de reajuste discreto. Se ativa, o dentista mantém alguma tensão muscular dorsal; se passiva, ele encosta-se ao apoio lombar da cadeira. Os instrumentos deverão estar colocados no ambiente de trabalho, de forma que os membros superiores os abarquem, sem alterar a postura neutra ${ }^{13}$. 
O uso de sistemas de ampliação pode alterar significativamente a postura, quer positiva, quer negativamente ${ }^{13,14}$.

O trabalho estático pode ser mais cansativo e doloroso que o dinâmico devido ao suprimento de oxigénio, ou seja, pode ser necessário que até metade dos músculos esteja simetricamente contraída. Para evitar a fadiga muscular, recomenda-se que o profissional alterne entre posturas balanceadas neutra e ativa, bem como fazer pequenas pausas que permitam levantar e andar ou sentar, alternando também marcações mais demoradas com outras mais breves ${ }^{13}$.

As posições desadequadas mais frequentemente reportadas são a flexão e rotação excessiva da cabeça do dentista, rotação do tronco, abdução dos membros superiores sem suporte e ângulo entre as coxas e os pés inferior a $90^{\circ 13}$.

Para além da ergonomia, também a ginástica laboral (sobretudo exercícios de alongamento) poderá contribuir para prevenir e atenuar o problema ${ }^{5}$.

\section{Ruído}

Neste setor profissional há exposição relevante ao ruído ${ }^{5,15}$.

Numa amostra de dentistas brasileiros 55\% apresentava alguma deficiência auditiva e $50 \%$ referia ter acufenos. Geralmente a diminuição da acuidade começa para as frequências mais elevadas (sobretudo entre 3000 e $6000 \mathrm{Hzs})^{15}$. Alguns autores recomendam não só o audiograma periódico, como sugerem o uso de proteção auricular ${ }^{5}$.

Os instrumentos de trabalho que apresentam níveis mais elevados de decibéis foram a turbina de alta rotação e o compressor; também foram mencionados as canetas odontológicas, o compressor de ar, o aspirador, o amalgamador e os próprios aparelhos de climatização ${ }^{5}$.

\section{Agentes químicos}

O mercúrio é um metal líquido, com evaporação a partir de $12^{\circ} \mathrm{C}$; a principal via de entrada é por isso a respiratória; $80 \%$ do mercúrio inalado é retido no organismo ${ }^{16}$. Os profissionais deste setor contactam com este agente sobretudo via inalatória através da produção de vapores (na preparação do produto, restauração e remoção da amálgama ${ }^{17}$ ), bem como na limpeza dos utensílios utilizados. Em função do número de procedimentos realizados, verificou-se que em serviços dentários especializados a exposição é superior quando comparados com serviços médicos dentários generalistas $^{18}$. Acredita-se que este agente consiga chegar à placenta ${ }^{17}$ e ao sistema nervoso central ${ }^{5,17}$.

Em países não desenvolvidos é frequente encontrar níveis de mercúrio mais elevados em dentistas e seus assistentes, quando comparados com a população geral. Este foi usado até há cerca de dois séculos como amálgama dentária, para restauração, por vezes em concentração que ia até os $50 \%$ (juntamente com prata, cobre e zinco). 
Existiam outras substâncias que se poderiam usar; mas o baixo custo, a facilidade de manuseamento e a durabilidade favoreceram durante algum tempo esta substância. Aliás, acredita-se que até $70 \%$ deste agente encontrado na água em circulação é proveniente dos consultórios dentários, sobretudo em países menos desenvolvidos. Para além disso, poderá haver ainda o efeito cumulativo para estes profissionais associado às suas próprias amalgamas ${ }^{17}$. A toxicidade do mercúrio depende da dose e de diversas caraterísticas metabólicas $^{18}$.

Os álcoois, por sua vez, são inflamáveis e podem levar a irritabilidade ocular e nasal, tosse, asma, cefaleia, tremor, depressão do sistema nervoso central e alterações cutâneas $^{16}$.

O éter é um irritante respiratório/ nasal, acima de 200 a 4000 ppm e extremamente inflamável; pode também ocasionar anorexia, cefaleia, tontura, sonolência, vómitos, bradicardia, sialorreia e alterações cutâneas ${ }^{16}$.

O latex das luvas de borracha poderá induzir dermatite de contato ou asma ${ }^{16}$.

O gluteraldeído e o formol foram relacionados com reações alérgicas, irritação ocular, náusea e cefaleia ${ }^{16}$; bem como alterações cutâneas, irritação nasal, tosse, pieira, asma, dispneia, patologia oncológica e queimadura, se procurarmos também em motores de pesquisa generalista.

Há eventual contato com metil-metacrilato (MMA) devido às bases de resina acrílica onde são montados os dentes artificiais; este pode causar irritação ocular e das mucosas, asma, enfizema, alterações no sistema nervoso periférico e alterações hepáticas ${ }^{16}$.

Existe também sílica em vários materiais usados neste setor (cerâmica, gesso, resinas, abrasivos); esta pode levar a silicose, cancro dos brônquios/ pulmão ou insuficiência cardíaca direita ${ }^{16}$.

O alginato é um dos materiais de moldagem mais prevalentes; por vezes são adicionados zinco, cádmio, chumbo e fluoretos. Este poderá contribuir para reações alérgicas e/ou alterações pulmonares ${ }^{16}$; bem como alterações cutâneas e oculares.

O cimento de vidro é constituído por ácido poliacrílico e é usado na restauração de dentes posteriores; pode levar a eczema de contato ${ }^{16}$; bem como alterações cutâneas (queimadura), oculares e também a nível das mucosas.

Substâncias adesivas como o BIS-GMA (1:2 bisfenol-A diglicil éter e ácido metacrílico), TEGMA (trietilenoglicoldimetacrilato), HEMA |2-dimetaciloiloxietil-2,2,4 (3,3,5)-trimetil hexametileno dicarbamatol apresentam potencial citotóxico. Em motores de busca generalista também se encontram referências a situações de lipotímia, sonolência, vertigem, cefaleia; alterações cutâneas (edema, prurido, eritema); tosse, rinorreia, rouquidão, odinofagia e alterações oculares (eritema, edema, prurido, lacrimejo e diminuição da acuidade). Os monómeros acrílicos (acrilatos, metacrilatos e acrilato epóxi) são usados neste setor para a elaboração de próteses e reconstrução dentária. 
Todos estão associados a eczema de contato alérgico. Por exemplo, na Finlândia, concluiu-se que estes eram os principais agentes a conseguir tal entre os profissionais deste setor; a reatividade cruzada não é rara ${ }^{16}$.

Os compostos libertadores de cloro (com destaque para o hipoclorito de sódio, mais no formato líquido) são utilizados a nível de desinfeção de superfícies inertes, ainda que possam causar reações cutâneas (queimadura, necrose, eritema), oculares (eritema, queimadura) e respiratórias ${ }^{16}$ (tosse, rinorreia, odianofagia, dispneia, broncoespasmo); bem como cefaleia, tonturas e náusea.

O paramonoclorofenol canforado (PMCC) é bactericida; contudo, pode libertar cloro e formol (este último pode causar ulcerações, queimaduras e despigmentação) ${ }^{16}$.

O EDTA (ácido etilenodiamino tetra-acético) tem caraterísticas desmineralizantes; contudo, pode causar irritação cutânea, ocular e respiratória; alguns investigadores consideram que há teratogenicidade, pelo menos em ratos ${ }^{16}$.

O ácido fosfórico é corrosivo para a pele (queimadura), olhos e mucosas; pode também induzir reações alérgicas ${ }^{16}$; bem como tosse, dispneia, conjuntivite, amaurose e convulsões.

Um estudo brasileiro comparou os conhecimentos relativos aos agentes químicos utilizados, entre dentistas-cirurgiões e estudantes da área; globalmente concluiu que ambos os grupos apresentavam noções limitadas ${ }^{16}$.

\section{Radiações}

Quase não se encontraram referências específicas a este item neste setor profissional, entre os artigos selecionados; a exceção foi dada à radiação ionizante dos $\mathrm{Rxs}^{5}$. Ainda assim os autores gostariam de salientar algumas noções gerais inseridas num artigo também publicado nesta revista ( $1^{\circ}$ volume), intitulado "Riscos Laborais associados às Radiações", do qual salientam os seguintes parágrafos.

Numa extremidade do espetro das radiações temos as de baixa frequência, pouca energia e mais abundantes (provenientes de computadores e telemóveis), não têm capacidade de danificar o material genético e são por isso designadas de não ionizantes; as radiações com mais energia são capazes de ionizar (ou seja, remover parte do átomo), como é o caso dos Rxs.

Os primeiros estudos sobre a radiação eletromagnética na saúde surgiram na década de 50; dentro dos efeitos agudos destacam-se as alterações de memória e na aprendizagem, segundo alguns autores; no contexto dos efeitos crónicos podem surgir leucemia ou outros cancros, alteração da fertilidade e do normal desenvolvimento da gravidez, bem como distúrbios imunológicos, neurológicos e/ ou cardíacos. Mesmo realizando a pesquisa em bases de dados conceituadas, encontram-se artigos com conclusões totalmente díspares, ou seja, existem autores que defendem a inexistência de qualquer risco comprovado; enquanto outros acreditam no oposto e, por fim, existem também investigações que alertam justamente para tal situação, ou seja, existência de 
resultados contraditórios, pelo que não se pode afirmar nada em concreto, com evidência científica clara e irrefutável. Uma parte considerável dos estudos é realizada com base em experiências em animais, pelo que extrapolar as conclusões para os humanos poderá não ser fácil ou linear. Ainda assim, a IARC (International Agency for Research on Cancer) classifica as radiações eletromagnéticas como "possivelmente carcinogénicas para os humanos". Contudo, considera-se que, dentro da população global, existirão indivíduos com suscetibilidades diferentes para a radiação.

Quanto à radiação ionizante muitos dos artigos consultados mencionam estudos efetuados nos sobreviventes das bombas atómicas, tentando extrapolar as conclusões para exposições a radiações de menor intensidade, mas de forma mais contínua. Também existem estudos sobre os trabalhadores de centrais nucleares, expostos ou não a acidentes. Por vezes, também se tenta extrapolar as conclusões obtidas em estudos com animais ou quimioterápicos mas, na realidade, sabe-se muito pouco sobre as consequências da radiação menos intensa e prolongada no tempo, como é o caso dos profissionais de saúde.

Acredita-se que a radiação associada aos procedimentos médicos constitua $95 \%$ da produzida pelo homem. Apesar de a evolução tecnológica ter permitido diminuir a radiação que estes profissionais são expostos por cada exame executado, ainda assim os procedimentos são efetuados com frequência crescente e, como a radiação é invisível, inodora e indolor, alguns profissionais banalizam a sua importância.

Genericamente, a intensidade de radiação recebida pelo profissional depende do tipo de equipamento médico utilizado, complexidade do procedimento, distância da fonte, tamanho do paciente, EPI (equipamentos de proteção individual) utilizados ou barreiras móveis (sendo estas uma das medidas mais eficazes). Por vezes, alguns profissionais evitam usar os EPI não só pelo desconforto, mas também pelo facto de argumentarem que, nessas condições, trabalharão mais lentificados, o que poderá aumentar o tempo de exposição às radiações; para além disso, uma parte significativa da radiação que atinge o profissional de saúde é proveniente da reflexão do doente.

\section{Stress}

Segundo alguns investigadores, o stress ocupacional destes profissionais poderá resultar da junção entre a existência de posturas forçadas e/ou mantidas (e eventuais sintomas), tarefas exigentes e/ou muito precisas ${ }^{5,19}$, riscos biológicos ${ }^{19}$ e com o facto de ter de lidar diretamente com o cliente (e por vezes também com a ansiedade destes) ${ }^{5,19}$; para além da responsabilidade, turnos prolongados e agentes químicos ${ }^{20,21}$.

A síndroma de Burnout resulta do stress crónico e é composta por três dimensões: a exaustão emocional, a descrença/ despersonalização e a baixa realização/ eficácia profissional; com repercussões a nível de presenteísmo, produtividade e qualidade de vida (global e laboral) ${ }^{20}$. 
O stress poderá ser atenuado pelo empregador se a remuneração for justa e se proporcionar alguma autonomia, progressão na carreira, segurança no vínculo laboral e condições de segurança no trabalho ${ }^{21}$.

\section{Satisfação Laboral}

A satisfação laboral neste setor está sobretudo associada à relação com o paciente; outros aspetos relevantes são a perceção sobre a remuneração, tempo disponível para questões pessoais, horário de trabalho, colegas de trabalho e existência de formação especializada ${ }^{22}$.

Num estudo brasileiro neste setor, por exemplo, apenas $12 \%$ da amostra estudada se englobou no patamar de "boa qualidade de vida"21.

\section{Medidas de proteção coletiva e individual}

Nenhum dos artigos selecionados mencionou de forma direta medidas de proteção coletiva. Contudo, poder-se-ão destacar neste contexto o uso de agentes químicos o menos tóxicos possíveis, um bom desenho ergonómico do posto de trabalho, utilização de equipamentos que emitam o menor ruído e vibração possível, rotatividade de tarefas e formação adequada aos profissionais relativa aos fatores de risco e riscos laborais.

A nível de equipamentos de proteção individual são mencionados o uso de bata de manga comprida, viseira ou óculos, gorro e luvas de latex ${ }^{3,23}$ (trocadas a cada hora de trabalho, se ainda estiver com o mesmo paciente ou entre cada paciente, obviamente). Antes de colocar as luvas devem-se lavar as mãos para diminuir a flora; depois de as tirar devem lavar-se novamente, bem como antes e depois de ir à casa de banho. Não esquecendo de limpar bem as unhas com escova adequada e usar produto antibacteriano em conjunto com a água; as unhas deverão estar limpas, curtas e não pintadas; alianças e anéis devem ser evitados. A secagem deve ser feita com toalhetes de papel ${ }^{23}$.

\section{Sinistralidade}

Os acidentes de trabalho neste setor justificam-se sobretudo pelo campo de trabalho pequeno, uso de instrumentos cortantes e/ou que trabalham a alta velocidade ${ }^{2,3,12}$, sendo razoavelmente frequente a criação de aerossóis e salpicos, bem como pela proximidade física com o cliente e/ou os movimentos bruscos que este possa ter ${ }^{2,12}$.

A parte do corpo geralmente mais atingida com acidentes por corto-perfurantes é o dedo; as agulhas nunca devem ser recapsuladas com a outra mão a segurar a tampa, nem as agulhas devem ser reutilizadas, encurvadas ou partidas, obviamente ${ }^{2}$.

\section{Doenças Profissionais}

Nenhum dos artigos consultados deu particular destaque para esta questão; contudo, são de realçar a este nível a patologia músculo-esquelética variada, doenças infeciosas (HIV, hepatites B e C, sobretudo), questões oncológicas diversas e, ao nível emocional, 
ansiedade, depressão e burnout.

\section{CONCLUSÃO}

Nenhum dos artigos selecionados mencionou de forma direta medidas de proteção coletiva. Contudo, poder-se-ão destacar neste contexto o uso de agentes químicos o menos tóxicos possíveis, um bom desenho ergonómico do posto de trabalho, utilização de equipamentos que emitam o menor ruído e vibração possível, rotatividade de tarefas e formação adequada aos profissionais relativa aos fatores de risco e riscos laborais.

A nível de equipamentos de proteção individual são mencionados o uso de bata de manga comprida, viseira ou óculos, gorro e luvas de latex.

Os acidentes de trabalho neste setor justificam-se sobretudo pelo campo de trabalho pequeno, uso de instrumentos cortantes e/ou que trabalham a alta velocidade, sendo razoavelmente frequente a criação de aerossóis e salpicos, bem como pela proximidade física com o cliente e/ou os movimentos bruscos que este possa ter. A parte do corpo geralmente mais atingida com acidentes por corto-perfurantes é o dedo; as agulhas nunca devem ser recapsuladas com a outra mão a segurar a tampa, nem as agulhas devem ser reutilizadas, encurvadas ou partidas.

Nenhum dos artigos consultados deu particular destaque para as doenças profissionais; contudo, são de realçar a este nível a patologia músculo-esquelética variada, doenças infeciosas (HIV, hepatites B e C, sobretudo), questões oncológicas diversas e, ao nível emocional, ansiedade, depressão e burnout.

Seria pertinente desenvolver investigações que avaliassem a realidade nacional.

\section{CONFLITOS DE INTERESSE, QUESTÕES ÉTICAS E/OU LEGAIS}

Nada a declarar.

\section{AGRADECIMENTOS}

Dr João Miranda de Azevedo, Médico Dentista, pelo serviço de consultadoria prestado relativamente aos dados recolhidos na revisão bibliográfica.

\section{BIBLIOGRAFIA}

1)Cheng $\mathrm{H}$, Su $\mathrm{C}$, Yen A, Huang $\mathrm{C}$. Factors affecting occupational exposure to needlestick and sharps among dentists in Taiwan: a nationwide survey. Plos ONE, 2012, $7(4), 1-7$.

2)Martins A, Pereira R, Ferreira R. Compliance with occupational post-exposure protocol for injuries among dental surgeons. Revista de Saúde Pública. 2010, 44(3), 1-12.

3)Garcia L, Blank V. Prevalência de exposições ocupacionais de cirurgiões-dentistas e auxiliares de consultório dentário e material biológico. Cadernos de Saúde Pública. 2006, 22(1), 97-108. 
4)Vasquez- Mayoral F, Sánchez-Pérez L, Olguín- Barreto Y, Acosta- Gío A. Dental school deans and dentists perceptions of infection control and HIV AIDS patient care: a challenge for dental education in Mexico. AIDS Patient Care and STDs. 2009, 23(7), 557562.

5)Nader H. Lesões por esforços repetitivos entre os cirurgiões-dentistas de RP-SP. Tese de Mestrado na Universidade de São Paulo, Escola de Enfermagem de Ribeirão Preto. 2006, 1-83.

6)Sakzewski L, Naser-ud-Din S. Work- related musculoskeletal disorders in Australian dentist and orthodontist: risk assessment and prevention. Work 52. 2015, 559-579.

7)Gutiérrez C, Portal G, Tejeda N, Tejeda I. Transtornos músculo esqueléticos y ergonomía en estomatólogos del municipio Sancti Spíritus, 2011. 2013, 15(19), 1-8.

8)Hayes M. A systematic review of musculoskeletal disorders among dental professionals. International Journal of Dental Hygiene. 2009,7, 159- 165.

9)Custódio R, Silva C, Brandão J. Ergonomic work analysis applied to dentistry- a brasilian case study. Work 41. 2013, 690-697.

10)Alto-Korte K, Alanko K, Kuuliala O, Jolanki R. Methocrylate and acrylate allergy in dental personnel. Contact Dermatitis. 2007, 57, 324-330.

11)Ohlendorf D, Erbe C, Hauck I, Nowak J, Hermanns I, Ditchen D et al. Kinematic analysis of work-related musculoskeletal loading of trunk among dentists in Germany. BMC Musculoskeletal Disorders. 2016, 17 (427), 1-11.

12)Ribeiro P, Brevidelli M, Tipple A, Ribeiro R, Gir E. Organizational safety climate and adherence to standard precautions among dentist. Acta Paulista de Enfermagem. 2013, 26(2), 192-197.

13)Pirvu C, Patrascu I, Pirvu D, Ionescu C. The dentist's operating posture ergonomic aspects. Journal of Medicine and Life. 2014, 7(2), 177-182.

14)Haes M, Osmotherly P, Taylor J, Smith $D$, Ho A. The effect of wearing loupes on upper extremity musculoskeletal disorders among dental hygienists. International Journal of Dental Hygiene. 2014,12, 174-179.

15)Gambarra $P$, Valença $A$, Rocha $A$, Cunha $D$. as repercussões do ruído ocupacional na audição dos cirurgiões-dentistas das unidades de saúde de família João Pessoa/ PB. Revista Brasileira de ciências da Saúde. 2012, 16(3); 361-370.

16)Arpone R, Teixeira A, Sitolino C, Pariri J, Nai G. Riscos ocupacionais químicos no conhecimento de cirurgiões-dentistas. Risco Ocupacional e Odontologia. Colloquium Vitae. 2012, 4(1), 38-51. 
17)Jamil N, Baquar M, Ilyas S, Qadir A, Arslan M, Salman M et al. Use of mercury in dental silver amalgam: an occupational and environmental assessment. Biomed Research International. 2016, 1-9.

18)Yilmaz H, Tutkun E, Demiralp K, Yilmar F, Aliyev V, Soylemezoglu T. Exposure to Mercury among dental health workers in Turkey: correlation with amalgam work and own fillings. Toxicology and Industrial Health. 2015, 31(10), ?-954.

19)Pozos- Radillo B, Preciado-Serrano M, Acosta-Fernandez M, Aguiterra M, PlascenciaCampos A. Síntomas psicofisiológicos preditores del estrés en odontólogos. Revista Médica del Instituto Mexicano del Seguro Social. 2016, 54(2), 151-158.

20)Zucoloto M, Jordani P, Bonafé F, Garcia P, Maroco J, Campos J. Síndrome de Burnout em cirurgiões dentistas com diferentes atuações profissionais. Psychology, Community \& Health. 2014, 3(2), 62-72.

21)Bueno R. Qualidade de vida dos cirurgiões-dentistas da rede pública dos municípios da AMFRI. Tese de Mestrado na Universidade Federal da Santa Catarina, Programa de Pós-graduação em Engenharia da Produção. 2004, 1-113.

22)Fahim A. Predictors of job satisfaction among practicing Dentists at hospitals in Suez Canal area, Egipt.International Journal of Occupational Medicine and Environmental Health. 2013, 26(1), 49-57.

23)Mejía G. Revision de las normas de bioseguridad en la atención odontologica con un enfoque em VIH/ SIDA. Universidade Odontológica. 2010, 29(62), 45-51.

Fluxograma da $1^{\text {a }}$ fase

Fluxograma da $2^{a}$ fase

\section{(1)Mónica Santos}

Licenciada em Medicina; Especialista em Medicina Geral e Familiar; Mestre em Ciências do Desporto; Especialista em Medicina do Trabalho; Presentemente a exercer nas empresas Medicisforma, Clinae, Servinecra, Serviço Intermédico, Securilabor, Gliese e CSW; Diretora Clínica da empresa Quercia; Diretora da Revista Portuguesa de Saúde Ocupacional on line. Endereços para correspondência: Rua Agostinho Fernando Oliveira Guedes, 42, 4420-009 Gondomar; s_monica_santos@hotmail.com.

\section{(2) Armando Almeida}

Doutorado em Enfermagem; Mestre em Enfermagem Avançada; Especialista em Enfermagem Comunitária; Pós-graduado em Supervisão Clínica e em Sistemas de Informação em Enfermagem; Docente na Escola de Enfermagem (Porto), Instituto 
da Ciências da Saúde da Universidade Católica Portuguesa; Diretor Adjunto da Revista Portuguesa de Saúde Ocupacional on line. 4420- 009 Gondomar. E-mail: aalmeida@porto.ucp.pt.

(3)Tiago Oliveira

Licenciado em Enfermagem pela Universidade Católica Portuguesa. Frequenta o curso de Técnico Superior de Segurança no Trabalho. Atualmente exerce a tempo inteiro como Enfermeiro do Trabalho. No âmbito desportivo desenvolveu competências no exercício de funções de Coordenador Comercial na empresa Academia Fitness Center, assim como de Enfermeiro pelo clube de futebol União Desportiva Valonguense. 4435-718 Baguim do Monte. E-mail: tiago_sc16@hotmail.com.

Santos M, Almeida A. Profissionais a exercer na Medicina Dentária: Principais Fatores de Risco e Riscos Laborais, Doenças Profissionais Associadas e Medidas de Proteção Recomendadas. Revista Portuguesa de Saúde Ocupacional on line. 2018, volume 5, S63-S65. DOI: 10.31252/RPSO.12.03.2018 